Original Research Paper

\title{
Prevalence and Clinical Correlates of COVID-19 Outbreak Among Health Care Workers in a Tertiary Level Hospital in Delhi
}

\author{
${ }^{1}$ Ankit Khurana, ${ }^{2}$ GP Kaushal, ${ }^{3}$ Rishi Gupta, ${ }^{4}$ Vansh Verma, ${ }^{4}$ Kabir Sharma and ${ }^{2}$ PM Kohli \\ ${ }^{1}$ Department of Orthopaedics, ESI Hospital, India \\ ${ }^{2}$ Department of Paediatrics, BSAMCH, India \\ ${ }^{3}$ Department of Biostatistics, Manokalp Clinic, Delhi, India \\ ${ }^{4}$ Medical Student, BSAMCH, India
}

Article history

Received: 16-04-2021

Revised: 31-05-2021

Accepted: 03-06-2021

Corresponding Author:

Ankit Khurana

Department of Orthopaedics, ESI

Hospital, India

Email: ankit24388@gmail.com

\begin{abstract}
COVID-19 has been shown to spread rapidly among Healthcare Workers (HCWs) and there is an urgent need to find and implement various mitigation strategies for these individuals. In this study, we summarize the epidemiological characteristics of the COVID-19 outbreak among HCWs in a tertiary care hospital and compare various parameters and preventive measures taken by infected and uninfected subjects. A retrospective questionnaire and record-based analysis was carried out to evaluate the epidemiological and clinical parameters of the participants. Preventive strategies such as masking, hand hygiene and use of prophylactic agents were compared for infected and uninfected subjects. Appropriate statistical tests were applied wherever required. The average age of participants in the COVID-19 positive group was 35.9 years and $52.1 \%$ of the positive HCWs were symptomatic. Three of these subjects needed hospitalization and one required admission to an intensive care unit. The study found a protective role of full course of prophylactic hydroxychloroquine in treated subjects versus untreated controls $(p=0.021)$ and benefit of N95 masks over others $(\mathrm{p}<0.001)$. Our results did not show any added protection with the use of other strategies (prophylactic vitamin C, D, zinc, betadine gargles, or other home remedies). We observed outbreak control with increased awareness, near universal testing, PPE provision, sanitation drive and promoting social distancing among HCWs. This study highlights the role of hydroxychloroquine prophylaxis, use of N95 mask and effect of early interventions in outbreak mitigation.
\end{abstract}

Keywords: COVID-19, Healthcare Workers, Hydroxychloroquine, N95 Mask, Infection Outbreak

\section{Introduction}

An epidemic caused by Severe Acute Respiratory Syndrome Coronavirus-2 (SARS-CoV-2), a positive sense single stranded RNA virus of zoonotic origin, emerged in Wuhan, Hubei Province, China, in December 2019. This infection has spread globally, causing significant morbidity and mortality. WHO declared it as a pandemic on 11th March 2020. Person-to-person transmission via droplet route is thought to be the most common mode of transmission as reported by WHO. The high secondary attack rate observed in COVID-19 makes way for explosive outbreaks and puts significant strain on healthcare services especially in developing nations like India.
Hence, early testing, contact tracing and isolation of cases is very important. Cases and deaths continue to rise as the disease becomes more widespread and more variants emerge. As of 26th May 2021. India is the second worst affected country in the world with nearly 27 million diagnosed cases causing more than 300,000 deaths and has become the primary cause of health-related concern in the country.

Optimum testing, usage of masks, contact tracing and isolation are the key interventions to control the spread of this illness. A prophylactic is highly desirable, especially in high risk groups such as healthcare workers. Low quality evidence exists for few drugs such as ivermectin and hydroxychloroquine but a significant benefit has not been demonstrated (Bartoszko et al., 2021). 
Healthcare workers are at a high risk of contracting and transmitting the disease because of significantly higher exposure as compared to the general population. OSHA has recognized COVID-19 as a hazard in this group. Epidemiological data regarding infections amongst healthcare is sparse from developing countries like India. Moreover, infections amongst healthcare workers reduces the available workforce, putting more strain on the healthcare system. Governments and health bodies worldwide have been working on pandemic mitigation strategies. These efforts aim to ensure rapid evaluation and care of patients, limiting further transmission and to better understand risk factors for transmission. With an aim to understand the prevalence and correlates of this infection in a tertiary level hospital in Delhi via a questionnaire based survey. We aim to look at the infection rate, efficacy and usage of masks, prophylactic measures being used by the healthcare workers and the various factors associated with a positive COVID-19 result, which may help in formulating better strategies to help prevent contracting infection at the workplace.

\section{Methods}

The study was carried out at a tertiary care hospital in New Delhi, India. A questionnaire based analysis was carried out to analyze the epidemiological and clinical parameters of healthcare workers of our hospital who had tested positive for COVID-19. A matched cohort of healthcare workers who tested negative was taken as the control group. Data collection was done by telephonic surveys, as well as evaluation of health records during the months of April-May2020. Responses were recorded via text, or in-person in certain cases, as the situation allowed. Various epidemiological parameters along with symptoms, comorbidities and preventive strategies adopted by healthcare workers were recorded after obtaining due consent. A similar survey was done for the control group (Fig. 1).

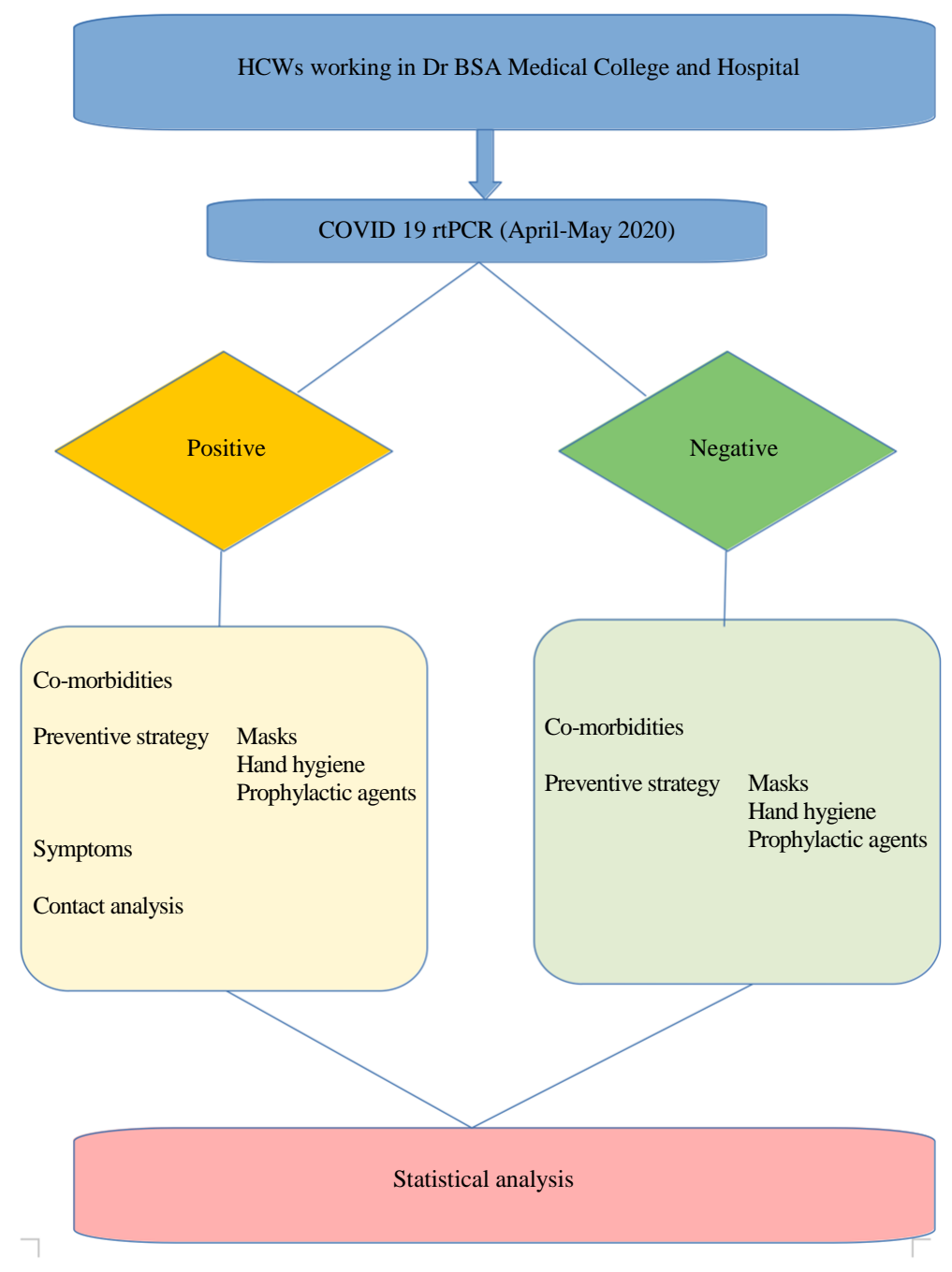

Fig. 1: Flowchart describing the experimental strategy used in present study 


\section{Statistical Analysis}

Data was coded and recorded in the MS Excel spreadsheet program. SPSS v23 (IBM Corp.) was used for statistical analysis. Normal distribution of data was assessed using the Shapiro-wilk test. Descriptive statistics were elaborated in the form of means for continuous variables and frequencies and percentages for categorical variables. Group comparisons for continuously distributed data were made using an independent sample ' $t$ ' test when comparing two groups. If data were found to be non-normally distributed, appropriate non-parametric tests were used for these comparisons. Chi-squared test was used for group comparisons for categorical data. In case the expected frequency in the contingency tables was found to be $<5$ for $>25 \%$ of the cells, Fisher's Exact test was used instead. Statistical significance was kept at $\mathrm{p}<0.05$.

\section{Ethical Approval}

Data collection and analysis of cases was part of a continuing public health outbreak investigation and was thus considered exempt from institutional review board approval. However, permission was sought from the head of the institute before the start of the study.

\section{Results}

\section{Demographic Data of Health Care Workers}

Males constituted $59.6 \%$ of total patients in the COVID positive group as opposed to $69.0 \%$ in the negative group. Mean age of the participants in the positive group was 35.98 years while in the negative group was 34.28 years. $16 \%$ of positive subjects in this study were doctors (including faculty and residents), $43.6 \%$ were nursing officers, $29.8 \%$ were paramedical staff (including sanitary workers, housekeeping staff and orderlies) and $10.6 \%$ security guards. There was no statistically significant difference in terms of age $(\mathrm{P}=$
$0.231)$, gender $(P=0.188)$ and designation $(P=0.102)$ between the study and the control groups (Table 1).

\section{Clinical Presentation}

$52.1 \%$ of participants in the positive group were symptomatic. Fever was the most common symptom, as experienced by $30.9 \%$ participants. Other commonly reported symptoms were sore throat, myalgia, headache and cough as reported by 20.2, 20.2, 14.9 and $11.7 \%$ of patients respectively. Rhinorrhea, shortness of breath, anosmia, nausea, pain abdomen were less common and reported by $4.3,6.4,3.2,2.1$ and $2.1 \%$ patients respectively. 1 patient each had mucosal dryness, hemoptysis, dysgeusia and loss of appetite (Table 2). Three patients in total needed hospitalization and only one of them needed intensive care for recovery. No mortality was observed in this study sample.

\section{Contact Analysis}

The mean number of close contacts per workday ( $>6$ hours) in the COVID Positive group was 19.13 and in the Negative group was 17.85. Close contacts per workday in the COVID Positive were as many as150compared to upto60 in the COVID negative/unknown group However, the difference was not statistically significant. $(\mathrm{W}=3794.500, \mathrm{p}=0.402)$. None of the participants who tested positive had suspected contact at home (Table 3).

\section{Comorbidity Analysis}

$10.6 \%$ (14/94) participants in the positive group reported chronic medical conditions, while $12.6 \%$ (14/87) of the participants in the control group had chronic medical conditions. The difference between the two groups was not statistically significant $(\mathrm{p}=$ 0.955 ) using a non-parametric test (Wilcoxon-MannWhitney U Test). The total number of co-morbidities in the positive ranged from $0-3$ while in the negative group from 0-4 (Table 4).

Table 1: Depicting demographic data of included healthcare workers

\begin{tabular}{|c|c|c|c|}
\hline \multirow[b]{2}{*}{ Parameters } & \multicolumn{2}{|l|}{ COVID } & \multirow[b]{2}{*}{$\mathrm{P}$ value } \\
\hline & Positive $(n=94)$ & Negative $(\mathrm{n}=87)$ & \\
\hline Age & $35.98 \pm 9.28$ & $34.28 \pm 8.97$ & 0.231 \\
\hline Gender & & & 0.188 \\
\hline Male & $56(59.6 \%)$ & $60(69.0 \%)$ & \\
\hline Female & $38(40.4 \%)$ & $27(31.0 \%)$ & \\
\hline Designation & & & 0.102 \\
\hline Doctor & $15(16.0 \%)$ & $9(10.3 \%)$ & \\
\hline Nursing Staff & $41(43.6 \%)$ & $41(47.1 \%)$ & \\
\hline Paramedical Staff & $28(29.8 \%)$ & $28(32.2 \%)$ & \\
\hline Security Guard & $10(10.6 \%)$ & $10(11.5 \%)$ & \\
\hline
\end{tabular}


Table 2: Clinical presentation of COVID positive healthcare workers

Frequency among COVID positive healthcare workers

Symptomatic

Yes

$49(52.1 \%)$

No

$45(47.9 \%)$

Maybe

$0(0.0 \%)$

Fever

$29(30.9 \%)$

Cough

$11(11.7 \%)$

Sore throat

$19(20.2 \%)$

Runny nose/Rhinorrhea

$4(4.3 \%)$

Mucosal Dryness

$1(1.1 \%)$

Hemoptysis

$1(1.1 \%)$

Breathlessness/ pneumonia/ chest congestion

Chest pain

Bodyache/Myalgia/ arthralgia/fatigue/weakness/malaise

Pain abdomen

Headache

Dysphagia

$1(1.1 \%)$

Diarrhea

Anosmia

Loss of appetite

Loss of taste/altered taste

Nausea/vomiting

Table 3: Contact analysis for COVID positive and control group

\begin{tabular}{llll}
\hline & COVID positive & COVID positive & P value \\
\hline Close contacts per workday $(>6 \mathrm{~h})$ & $19.13 \pm 21.50$ & $17.85 \pm 13.70$ & 0.402 \\
Place of suspected contact & & & - \\
Home & $0(0.0 \%)$ & - & \\
Hospital & $94(100.0 \%)$ & - & - \\
Duration of contact & $32(51.6 \%)$ & - \\
$<6 \mathrm{~h}$ & $30(48.4 \%)$ & $\mathrm{h}$ & \\
\hline 6
\end{tabular}

Table 4: Distribution of chronic medical conditions among positive healthcare workers and control group

\begin{tabular}{|c|c|c|c|}
\hline & COVID positive & COVID negative & $\mathrm{P}$ value \\
\hline Chronic Medical Condition & & & 0.955 \\
\hline Yes & $14(10.6 \%)$ & $14(12.6 \%)$ & \\
\hline No & $82(87.2 \%)$ & $75(86.2 \%)$ & \\
\hline Maybe & $2(2.1 \%)$ & $1(1.1 \%)$ & \\
\hline Diabetes & $3(3.2 \%)$ & $3(3.4 \%)$ & \\
\hline HTN & $7(7.4 \%)$ & $4(4.6 \%)$ & \\
\hline Hypothyroidism & $2(2.1 \%)$ & $1(1.1 \%)$ & \\
\hline Asthma & $1(1.1 \%)$ & $4(4.6 \%)$ & \\
\hline Pregnancy & $1(1.1 \%)$ & $0(0.0 \%)$ & \\
\hline Others & $1(1.1 \%)$ & $2(2.3 \%)$ & \\
\hline
\end{tabular}

\section{Prophylactic Agents used by Healthcare Workers}

Chi-squared test was used to determine the association between 'COVID positivity' and 'prophylactic hydroxychloroquine intake'. There was a statistically significant difference between the various groups in terms of distribution of prophylactic hydroxychloroquine intake $\left(X^{2}=17.159, \mathrm{p}=<0.001\right)$. $18.4 \%$ of the participants in the COVID negative group had taken a full course ( 7 weeks or more) of hydroxychloroquine prophylaxis as opposed to $6.4 \%$ in the COVID positive group an analysis of those who had taken full course as compared to those who had taken either incomplete course or had not taken at all revealed a statistically significant difference with $\mathrm{p}=0.021 \mathrm{using}$ the Fisher's exact test (Table 5 and 6).

Other Commonly used prophylactic agents by participants in this study included Betadine gargles, Vitamin C, D, Zinc, saline gargles and other home remedies did not reveal a statistically significant association with 
COVID status among healthcare workers ( $p>0.05)$. 30.9\% of the participants in the positive group used prophylaxis other than hydroxychloroquine while in the negative group $23.0 \%$ of the participants used other prophylaxis (Table 5).

$95.7 \%$ of the participants in the COVID positive group used masks at both work and community. Similarly, $90.8 \%$ of the participants in the control group had mask usage at both places. Chi-squared test was used to explore the association between 'COVID status' and place of mask usage. The difference was statistically insignificant $\left(X^{2}=\right.$ 1.781, $\mathrm{p}=0.182$ ). Chi-squared test was further used to explore the association between 'COVID' and 'Minimum level of protection'. A statistically significant difference was observed in terms of minimum level of protection $\left(X^{2}\right.$ $=15.668, \mathrm{p}=<0.001)$. In the COVID positive group, $30.9 \%$ of the participants had a minimum level of protection as an N95 mask, 63.8 and $5 \%$ of the participants had minimum level of protection as 3-Ply Mask and bandana respectively. The percentages in the control group were 57.5, 34.5 and $8.0 \%$ for N95, 3ply and Bandana mask, respectively. Thus, the control group had a larger fraction of people using N95 as compared to 3 ply and bandana $(\mathrm{p}<0.001)$, thus a significantly higher number of participants were using N-95 masks as compared to bandana in the control group.
Average days after which the N95 mask was changed was not normally distributed in the 2 groups. Thus, a non-parametric test (Wilcoxon-Mann-Whitney U Test) was used for comparison. The mean (SD) number of days before the mask was changed in the COVID Positive group was 7.81 (10.53) while in the negative group was 7.33 (9.23). No significant difference was observed between the two groups in terms of N95 mask change frequency $(\mathrm{W}=3821.500, \mathrm{p}=0.439)$ (Table 7 and 8).

\section{Hand Hygiene Practices among Health Care Workers}

Participants in the COVID positive group had a larger proportion of people who washed hands for over $20 \mathrm{sec}$ than the control group, but the difference was not statistically significant. The variable Hand Washing/Sanitizer-Use Frequency (Times/day) was not normally distributed in the two groups. Thus, a nonparametric test (Wilcoxon-Mann-Whitney U Test) was used to make comparisons. The mean (SD) of Handwashing/Sanitizer-Use Frequency (Times/day) in the positive group was 20.66 (12.79) as compared to 18.03(13.16) in the control group. There was no significant difference between the two groups in terms of Handwashing/Sanitizer-Use Frequency (Times/day) (W $=4633.000, \mathrm{p}=0.120)($ Table 9$)$.

Table 5: Analysis of preventive and prophylactic strategies adopted by healthcare workers

\begin{tabular}{|c|c|c|c|}
\hline & COVID Positive & COVID negative & $\mathrm{P}$ value \\
\hline Hydroxychloroquine Intake & & & $<0.001$ \\
\hline Full course ( 7 weeks $)$ & $6(6.4 \%)$ & $16(18.4 \%)$ & \\
\hline Partial course & $48(51.1 \%)$ & $20(23.0 \%)$ & \\
\hline Not taken & $40(42.6 \%)$ & $51(58.6 \%)$ & \\
\hline Prophylaxis other than hydroxychloroquine (Yes) & $29(30.9 \%)$ & $20(23.0 \%)$ & 0.234 \\
\hline Betadine gargles & & & 0.061 \\
\hline Not Taken & $84(89.4 \%)$ & $84(96.6 \%)$ & \\
\hline Taken & $10(10.6 \%)$ & $3(3.4 \%)$ & \\
\hline Vitamin C & & & 0.672 \\
\hline Not Taken & $82(87.2 \%)$ & $74(85.1 \%)$ & \\
\hline Taken & $12(12.8 \%)$ & $13(14.9 \%)$ & \\
\hline Vitamin D & & & 0.722 \\
\hline Not Taken & $89(94.7 \%)$ & $84(96.6 \%)$ & \\
\hline Taken & $5(5.3 \%)$ & $3(3.4 \%)$ & \\
\hline Zinc & & & 0.622 \\
\hline Not Taken & $91(96.8 \%)$ & $86(98.9 \%)$ & \\
\hline Taken & $3(3.2 \%)$ & $1(1.1 \%)$ & \\
\hline Ayurvedic/Home Remedies & & & 0.503 \\
\hline Not Taken & $86(91.5 \%)$ & $77(88.5 \%)$ & \\
\hline Taken & $8(8.5 \%)$ & $10(11.5 \%)$ & \\
\hline Saline/Hot Water Gargles & & & 0.685 \\
\hline Not Taken & $86(91.5 \%)$ & $81(93.1 \%)$ & \\
\hline Taken & $8(8.5 \%)$ & $6(6.9 \%)$ & \\
\hline
\end{tabular}

Table 6: Distribution of hydroxychloroquine prophylaxis usage among healthcare workers

\section{COVID status}

\begin{tabular}{llcl} 
Hydroxychloroquine & Negative & Positive & P value \\
\hline Full course & 16 & 6 & 0.012 \\
Not taken/Incomplete course & 71 & 88 & \\
\hline
\end{tabular}


Table 7: Analysis of mask usage among healthcare workers

\begin{tabular}{llll}
\hline & COVID positive & COVID negative & P value \\
\hline Mask use at work only) & $3(3.2 \%)$ & $7(8.0 \%)$ & 0.199 \\
Mask use in community only & $1(1.1 \%)$ & $1(1.1 \%)$ & 1.000 \\
Mask use at both places & $90(95.7 \%)$ & $79(90.8 \%)$ & 0.182 \\
N95 mask use (present) & $62(66.0 \%)$ & $77(88.5 \%)$ & $<0.001$ \\
3-Ply mask use (present) & $62(66.0 \%)$ & $34(39.1 \%)$ & $<0.001$ \\
Bandana use (present) & $5(5.3 \%)$ & $3(3.4 \%)$ & 0.722 \\
Minimum level of protection & $29(30.9 \%)$ & $50(57.5 \%)$ & $<0.001$ \\
N95 & $60(63.8 \%)$ & $30(34.5 \%)$ & \\
3-Ply mask & $5(5.3 \%)$ & $7(8.0 \%)$ & 0.439 \\
Bandana & $7.81 \pm 10.53$ & & $<0.001$ \\
Mask change frequency (no. of days each mask used) & & & \\
Mask use at time of contact & $58(61.7 \%)$ & & \\
Yes & $0(0.0 \%)$ & \\
No & $36(38.3 \%)$ &
\end{tabular}

Significant at $\mathrm{p}<0.05$

Table 8: Analysis of mask used as minimum level of protection among healthcare workers

\begin{tabular}{lc}
\hline Minimum level of protection & Adjusted p values \\
\hline N95 Vs. 3-Ply Mask & $<0.001$ \\
N95 Vs. Bandana & 0.757 \\
3-Ply Mask Vs. Bandana & 0.172
\end{tabular}

Table 9: Analysis of hand hygiene practices among health care workers

\begin{tabular}{llll}
\hline & COVID positive & COVID negative & P value \\
\hline Hand washing Technique & & & 0.243 \\
Normal $(<20 \mathrm{~s})$ & $30(31.9 \%)$ & $52(59.8 \%)$ & \\
Surgical (>20s) & $64(68.1 \%)$ & $35(40.2 \%)$ & \\
Handwashing/Sanitizer-Use Frequency (Times/day) & $20.66 \pm 12.79$ & $18.03 \pm 13.16$ & 0.120 \\
\hline
\end{tabular}

\section{Discussion}

We have provided an initial assessment of the epidemiology and risk mitigation dynamics of the COVID-19 outbreak among healthcare workers of our hospital. We observed a marked increase in positive cases within a few days, of which only three needed hospitalization and one required admission to intensive care unit. Only $52.1 \%$ of the positive healthcare workers showed symptoms. A possible reason for the same is believed to be the younger cohort of patients amongst the healthcare workers who got infected. Moreover, we found that the majority (about 60\%) of COVID-19 cases were male, although the reason remains to be clarified.

Findings of present study found some protective role of full course ( 7 weeks) of prophylactic hydroxychloroquine as compared to a control group of negative healthcare workers with $p=0.021$ and use of N95 masks over others. Our results did not show any added protection with the use of other strategies in the form of prophylactic Vitamin C, D, Zinc, betadine gargles, or any other home remedy.

\section{Infectivity and Transmission}

Estimation of the prevalence and transmission for undocumented novel Coronavirus (SARS-CoV-2) infections is critical for understanding the total prevalence and pandemic potential. Another factor is the mode of viral transmission through aerosol and fomites. SARS-CoV-2is viable for over $3 \mathrm{~h}$ in aerosol mode but titers decreased significantly after $3 \mathrm{~h}$ moreover, the virus is much more stable on stainless steel and plastic compared to copper and cardboard and can be obtained from steel and plastic surfaces even after 3 days (van Doremalen et al., 2020). However, till date there is no consensus on Airborne transmission as contradictory versions exist. The matter remains unresolved partially due to the difference in definitions of air borne transmission, different conditions used by various researchers and infectivity if Viral RNA particles in air (Ong et al., 2020). Lauer et al. (2020) took data of 181 patients and estimated that the median incubation period as 5.1 days which is similar to SARS and 2.5 times that of influenza virus. They found that less than $2.5 \%$ of cases became symptomatic within 2.2 days of exposure and $97.5 \%$ became symptomatic within 11.5 days of exposure. $1 / 100$ developed symptoms after 14 days of exposure.

SARS-CoV-2 shows high infectivity in the hospital setting. It has been isolated from sputum, nasopharynx, oropharynx, stool, blood and conjunctiva but not from urine, breastmilk, amniotic fluid and cord blood (Zou et al., 
2020; Pan et al., 2020a; Wang et al., 2020a; Liang and $\mathrm{Wu}, 2020)$. The highest load was found at onset of symptoms and $30 \%$ persisted to have RNA load detected by RT-PCR after 20 days of symptom onset (To et al., 2020). Rapid decline in viral load follows seroconversion which occurs at 6-12 days (Woelfel et al., 2020). Furthermore, Liu et al. (2020a) found that severe cases have higher viral loads and shed the virus longer explaining the high infectivity in hospital settings. Viral load is an indicator of disease severity. Cereda et al. (2020) found the pre-symptomatic individuals had viral loads comparable to symptomatic individuals with no significant statistical difference. Wang et al. (2020b) identified 55 family members of known COVID patients and found that RT-PCR positivity 1-7 days before symptom onset. Most patients who were likely to have mild symptoms were younger, which is similar to our series where the mean age is 35 years and only over $50 \%$ displayed any symptoms. Hu et al. (2020) found that truly "asymptomatic" individuals had a mean age of 14 years. This is in contrast to the diamond princess cruise ship cohort where the asymptomatic population which was RT-PCR positive was $17.9 \%$. However, patients were not followed long enough to assess how many of them were pre-symptomatic (Mizumoto et al., 2020). Asymptomatic transmission was reported early during the course of the epidemic (Bai et al., 2020; Qian et al., 2020). This could be a reason for rapid spread of the virus among healthcare workers, where a patient or co-workers can be asymptomatic carriers for COVID.

Du et al. (2020) published and evaluated 462 transmission events and serial interval (time between symptom onset between transmitter and transmitee) and found it to be 4 days and they reported $6 \%$ of transmissions are pre-symptomatic. Epidemic control becomes challenging due to undocumented infections. In China, as high as $86 \%$ of infections were undocumented during early epidemic which were either mild or asymptomatic. Undocumented infections were estimated to be half as infectious as symptomatic infections (Li et al., 2020). Environmental viability was assessed and 13 patients with mild COVID 19 were isolated in negative pressure rooms. The authors found viral RNA on cell phones with $85 \%$ positivity as well as toilets and air samples. 63\% of air samples were positive for RNA but the live virus could not be cultured. Hence hinting at regular aerosolization of the virus (van Doremalen et al., 2020). The possibility of airborne transmission adds complexity to infection control strategies especially in the setting of tertiary hospitals with a large number of positive patients.

Peak infectiousness occurs about 1 day before symptom onset. When viremia is assessed Viral loads as previously discussed the viral load is high even in the pre-symptomatic phase and peaks at symptom onset (Zhou et al., 2020). He et al. (2020) studied viral shedding by looking at 414 swabs from 94 patients of positive patients from symptom onset. None of them had severe symptoms on presentation although 18 developed the same during the course. They found viral load does not correlate with sex, severity and age. They also evaluated 77 detailed transmission pairs based on serial interval (that is time from symptom onset in transmitter to symptom onset in transmittee) and they estimated that infectiousness started at 2.3 days before symptom onset and peaked 0.7 days before symptom onset. Estimated pre-symptomatic transmission was $44 \%$ in their study. This is in line with the findings of Du et al. (2020) and Ferretti et al. (2020). The authors of this study also suggest that this percentage will vary depending on the efficacy of active case finding with those places with a high percentage of case finding to have a higher percentage of presymptomatic transmission. Huang et al. (2020) and Lin et al. found that higher viral loads are found in lower respiratory specimens in critically ill. Xiao et al. (2020) have found in their study of 56 patients that prolonged shedding of viral RNA can be seen in mild or moderate disease and found that Nasopharyngeal PCR remains positive a median of 24 days after symptom onset with a $5 \%$ positivity at 5 weeks, however its relation with infectiousness remains unknown. In mild cases, live virus is isolated up to day 8 after symptom onset. In a study from Taiwan, the authors found that no secondary cases were identified from exposures occurring after 5 days from symptom onset (Cheng et al., 2020). RNA detection by PCR maybe for much longer. For critical illnesses the scenario can be slightly different and there can be prolonged shedding of viral RNA lasting many weeks, hence leading to high infectivity from critically ill patients in the hospital (Arons et al., 2020).

\section{Clinical Features}

There is a spectrum of presentations for COVID-19 which include: Asymptomatic, mildly symptomatic, severe symptomatic with spontaneous recovery and severe symptomatic with development of an ARDS proinflammatory syndrome (Xiao et al., 2020; Siddiqi and Mehra, 2020; Pan et al., 2020b). Compiled series of hospitalized patients revealed fever and cough to be the most common symptoms followed by myalgias, fatigue, sore throat, nausea, vomiting, diarrhea, headache and rhinorrhea (Huang et al., 2020; Guan et al., 2020; Pan et al., 2020c) Gastrointestinal (GI) symptoms have been given more emphasis in recent data. Pan et al. (2020c) found that $42 \%$ had GI symptoms as part of their syndrome. Anosmia, hyposmia and dysgeusia have been reported as per society reports that state that anecdotal evidence is accumulating. A large-scale real-time symptom monitoring study seems to confirm the importance of anosmia/ageusia (Reinhard et al., 2020). Our findings are in line with available literature 
with most common symptoms being fever, followed by bodyache, sore throat, cough, headache, pain abdomen, anosmia and in more severe cases- breathlessness and need for mask ventilation.

\section{Comorbidity Evaluation}

Risk factors for severe disease as per several authors are comorbidities like hypertension, heart disease, diabetes, CKD, Pre-existing pulmonary disease. Jin and $\mathrm{Hu}$ (2021) found that diabetic patients were at greater risk for severe infections, poorer prognosis and higher mortality compared to patients without diabetes. Case fatality rate is defined as the number of COVID 19 deaths divided by the total number of infections. It is subject to bias if the number of cases tested is too low (upward bias) or when deaths have not yet occurred in the setting of ongoing illness (downward bias). Wu et al. (2021) used this information to assess age specific CFR as $1.4 \%$ with $0.3 \%$ under 30 and $2.6 \%$ over 59 and increasing precipitously as age increases. The present study did not find any severe illness and only 3/94 positive patients needed hospital admission. In contrast, the mean age of infected health care workers in our study was 35 years and only 14/94 (14.9\%) had one or more comorbidities.

\section{Hydroxychloroquine Prophylaxis}

The Indian Council of Medical Research, under the Ministry of Health and Family Welfare, has recommended chemoprophylaxis with hydroxychloroquine $(400 \mathrm{mg}$ twice on day 1 , then $400 \mathrm{mg}$ once a week thereafter for 7 weeks) for asymptomatic healthcare workers treating patients with suspected or confirmed COVID-19 and for asymptomatic household contacts of confirmed cases. Our results show a significant reduction in the rate of infection by taking full course of hydroxychloroquine prophylaxis, however accumulating therapeutic data shows no benefit. Hydroxychloroquine was found to have in vitro activity early on in the pandemic. It binds to ACE2 receptors and as a result may block viral entry and intracellular transfer (Chen et al., 2020a). An interim report with a rather uncertain methodology from China reported efficacy and safety in 100 patients leading to expert consensus in China to recommend chloroquine as the first line for all patients with COVID 19. Low quality RCTs from China showed no significant effect of HCQ with respect to negative throat swab, days to being afebrile and radiographic progression (Gao et al., 2020; Wang et al., 2020c). The bias was that both groups received other antivirals that are lopinavir and ritonavir. Chen et al. (2020b) in another RCT suggested superior clinical results in hydroxychloroquine treated individuals but the study has several biases in terms of 80 excluded patients for uncertain reasons and it is unclear how other standard therapies were distributed among hydroxychloroquine and non-hydroxychloroquine groups.
A small non-randomized french clinical trial (26 HCQ vs 16 HCQ) suggested faster viral clearance with hydroxychloroquine, but they excluded 6 patients from the analysis who were either shifted to ICU or got worse which suggests confounding in their data (Gautret et al., 2020a; 2020b) The society (International society of antimicrobial chemotherapy) that published this paper said in a statement that the published study did not meet the societies expected standard relating to a lack of better explanation of inclusion criteria.

The same group of French authors (Gautret et al., 2020b) have published synergy between azithromycin and hydroxychloroquine based on faster viral clearance when a combination of the two drugs was used. The limitations of their findings were - no control arm and only patients with mild disease were included. Lane et al. (2020) did a network cohort and self-controlled case series study which found that addition of azithromycin may induce heart failure and cardiovascular mortality, potentially due to synergistic effects on QT length. Mahevas et al. (2020) did a retrospective analysis of 181 patients from 4 hospitals of which 84 who had taken hydroxychloroquine and 79 did not. The authors found no benefit and approximately $10 \%$ needed to discontinue hydroxychloroquine due to $\mathrm{QT}_{\mathrm{C}}$ prolongation issue. Tang et al. (2020) conducted a multi-centric RCT of 150 hospitalized patients with COVID and found that hydroxychloroquine doesn't lead to faster viral clearance. Post hoc analysis controlling for use of other antivirals found early symptom relief with hydroxychloroquine and faster improvement in CRP. Magagnoli et al. (2020) retrospectively analyzed 368 males. They found that in their non-randomized study the risk of death was higher with the hydroxychloroquine group. Axfors et al (2021) conducted a meta analysis which found that treatment with hydroxychloroquine is associated with increased mortality in COVID-19 patients. However, their findings had unclear generalizability to children, pregnant women and people with comorbidities. In a review article, Juurlink (2020) emphasizes that use of hydroxychloroquine and azithromycin for COVID-19 prevention is currently supported by in vitro data and weak studies. Physicians should be aware of several uncommon but potentially life-threatening adverse effects before making any decisions.

\section{Mask Usage}

A recent study on influenza referenced above shows no difference lab confirmed influenza with the use of either N95 or surgical mask, influenza virus like COVID is an RNA virus with similar infectivity (Radonovich et al., 2019). Leung et al. (2020) assessed the use of masks in transmission of respiratory viruses including Influenza, non COVID Coronaviruses and Rhinoviruses between 2013 to 2016. They included 246 participants with respiratory symptoms who had confirmed respiratory 
virus and randomized 50\% to wear masks and assess exhaled breath particles and classified them as either droplets or aerosols. There was a significant decrease in the recovery of coronaviruses from droplets and aerosols with the use of masks. While this study was not done in the context of COVID 19 but the findings are extrapolatable to COVID patients especially those who are symptomatic.

We, however, found N95 to be more effective in preventing infection as compared to other masks ( 3 ply mask and cloth bandana). $(\mathrm{p}<0.001)$ There was no difference in frequency of N95 mask change among positive and control groups and most healthcare workers who tested positive did show good compliance with mask usage in the hospital as well as in the community.

\section{Outbreak Control}

\section{Situation Appropriate Personal Protective Equipment}

McMichael et al. (2020) explained transmission and outcomes in an epidemic at an unprepared long-term care facility. 81/130 residents, 34/170 health care workers and 14 visitors became positive. The median age of 81 residents was high with most having several co-morbidities and eventually, $27 \%$ died. The contributing factors analyzed by the authors were: Staff members worked while symptomatic, Staff members worked in more than one facility, Inadequate familiarity and adherence to the standard, droplet and contact precautions and eye protection recommendations, challenges to implementing infection control practices including inadequate supplies of PPE and other items (e.g., Hand sanitizer), delayed recognition of cases because of low index of suspicion, limited testing availability and difficulty identifying persons with COVID-19 based on signs and symptoms alone. The authors concluded that "Long-term care facilities should take proactive steps to protect the health of residents and preserve the health care workforce by identifying and excluding potentially infected staff members and visitors, ensuring early recognition of potentially infected patients and implementing appropriate infection control measures" (McMichael et al., 2020; Wang et al., 2020b). The hospital administration taking cognizance to the above research took proactive steps to contain the outbreak among health care workers. All health care workers who tested positive were immediately instructed to isolate themselves. Similarly, high risk contacts of positive individuals were sent on quarantine pro-actively and timely. Situation appropriate PPEs were provided and all health care workers were instructed to adhere to given rules for their protection. Surveillance was carried out and most of the 1605 health care workers employed at our facility were tested. This led to further case identification and lead to contact tracing, quarantine of close contacts and control of the outbreak.

\section{Movement Restriction}

Movement restrictions and mandatory social distancing are highly effective in controlling transmission and decrease transmission coefficient from 4 to 1.6 (and later less than 1 when quarantine is initiated) (Fang et al., 2020). The institute administration promoted mandatory social distancing and necessary quarantine/isolation in our mitigation strategy. This led to control of the initial outbreak among our health care workers. Lower case reporting was achieved subsequently.

\section{Contact Tracing}

Ferretti et al. (2020) studied the impact of 2 interventions which were contact tracing and case identification. Their model explains that if there is a delay in isolation and contact quarantine, there is less likelihood of it being effective at controlling the epidemic. The authors also support the use of a mobile phone app which is a form of digital contact tracing by keeping a temporary record of proximity events between individuals. This approach would not require coercive surveillance since the system can achieve epidemic suppression. This is a promising strategy. Epidemic control becomes feasible with contact tracing if the minimal delay can be achieved. The hospital administration's approach in the present scenario was comparable. Rapid contact tracing was initiated and all contacts and positive individuals were encouraged to download the government of India's contact tracing app (Arogya Setu) immediately when the outbreak was identified.

\section{Universal Testing Drive}

Gudbjartsson et al. (2020) found that targeted sampling was changed to random sampling in Ireland which was an open invitation to asymptomatic or mild cold symptoms. The authors found $<1 \%$ had positive SARS-CoV-2 PCR. Lavezzo et al. (2020) assessed the role of mass testing in a town in Italy with a population of 3000. The entire town was quarantined for 14 days after the index case had died. The researchers found a high rate of asymptomatic infection and transmission upon universal testing. Two PCR samples were taken 12 days apart and the authors found that 85 and $71 \%$ of the population took part in the survey. Prevalence decreased to $1.2 \%$ the second time from an initial $2.6 \%$. A quarter of those positive on the second time point were new infections which the researchers found were due to contact with asymptomatic infected individuals either before lockdown or within their household. This substantiates the point that asymptomatic/presymptomatic transmission plays a key role in the ongoing transmission of SARS-CoV-2. The effective reproductive number declined from 3 to 0.14 after the end of lockdown - effectively ending the outbreak. Studies with near - universal screening of various 
populations are now becoming more available, finding a wide range of asymptomatic people with positive PCR Pregnant women in NYC-13.5\% $(87 \%$ of total infections); homeless shelter in Boston - 36\% (a great majority of total infections); Town in Italy - <1\% (41\% of total infections); Iceland - <1\% (43\% of total infections) and cruise ship Diamond Princess - 9\% (46\% of total infections) (48-50) Thus, serological data shows SARS-CoV-2 has a significant iceberg effect. Our approach to control involved near universal testing of health care workers to understand the level of the outbreak and early outbreak control.

\section{Rigorous Sanitization Drive}

Liu et al. (2020b) measured viral RNA in various areas in 2 hospitals. They found that ventilation and sanitization played a significant role in the number of viral RNA copies detected. Ventilated areas had a lower RNA concentration as compared to unventilated areas like toilets. Also, a rigorous sanitization drive led to virtually undetectable detection of viral RNA. This led the hospital authorities to undertake a massive sanitization drive in the hospital.

\section{Strengths and Limitations}

Limitations of this study include its cross-sectional design, so associations between potential risk factors and outcomes of health and well-being may not be causal. In particular, participants with poorer well-being might differentially report superior preventive behaviors. We studied employees of a single institute, who may not be representative of other workforces.

Strengths of the study include its overall high response rate, examination of employees who are not directly involved in health care and workplace factors. We are conducting repeated surveys to track changes in individual health and well-being over time and to allow more robust causal inferences.

\section{Conclusion}

The transmission potential of COVID-19 is very high and the number of cases may become largely unsustainable for the healthcare system in a very shorttime horizon. It is important to protect the healthcare force for effective epidemic management. We observed outbreak control with increased awareness, near universal testing, PPE provision, sanitization drive and promoting social distancing among health care workers. This study also brings forth the role of hydroxychloroquine prophylaxis, use of N95 mask and effect of early interventions in outbreak mitigation. Aggressive containment strategies are required to control COVID-19 spread and catastrophic outcomes for the healthcare system in the absence of a therapy/vaccine to avoid overwhelming the critical care capacity of any healthcare facility.

\section{Author's Contributions}

Ankit Khurana: Participated in formulating the study, data collection, analysis and contributed to writing the manuscript.

GP Kaushal and PM Kohli: Concept and guidance.

Rishi Gupta: Biostatistician.

Vansh Verma and Kabir Sharma: Participated in data collection, analysis and contributed to writing the manuscript.

\section{Ethics}

This article is original and contains unpublished material. The corresponding author confirms that all of the other authors have read and approved the manuscript and no ethical issues involved.

\section{References}

Arons, M. M., Hatfield, K. M., Reddy, S. C., Kimball, A., James, A., Jacobs, J. R., ... \& Jernigan, J. A. (2020). Presymptomatic SARS-CoV-2 infections and transmission in a skilled nursing facility. New England Journal of Medicine, 382(22), 2081-2090. https://doi.org/10.1056/NEJMoa2008457

Axfors, C., Schmitt, A. M., Janiaud, P., van't Hooft, J., Abd-Elsalam, S., Abdo, E. F., ... \& Hemkens, L. G. (2021). Mortality outcomes with hydroxychloroquine and chloroquine in COVID-19 from an international collaborative meta-analysis of randomized trials. Nature Communications, 12(1), 1-13. https://doi.org/10.1038/s41467-021-22446-Z

Bai, Y., Yao, L., Wei, T., Tian, F., Jin, D. Y., Chen, L., \& Wang, M. (2020). Presumed asymptomatic carrier transmission of COVID-19. JAMA, 323(14), 1406-1407. https://doi.org/10.1001/jama.2020.2565

Bartoszko, J. J., Siemieniuk, R. A., Kum, E., Qasim, A., Zeraatkar, D., Ge, L., ... \& Brignardello-Petersen, R. (2021). Prophylaxis against covid-19: living systematic review and network meta-analysis. BMJ, 373, n949. https://doi.org/10.1136/bmj.n949

Cereda, D., Tirani, M., Rovida, F., Demicheli, V., Ajelli, M., Poletti, P., ... \& Merler, S. (2020). The early phase of the COVID-19 outbreak in Lombardy, Italy. arXiv preprint arXiv:2003.09320. http://arxiv.org/abs/2003.09320

Chen, J., Liu, D., Liu, L., Liu, P., Xu, Q., Xia, L., ... \& Lu, H. (2020a). A pilot study of hydroxychloroquine in treatment of patients with common coronavirus disease-19 (COVID-19). Journal of Zhejiang University (Medical Science), 49(1), 215-219. https://doi.org/10.3785/j.issn.1008-9292.2020.03.03 
Chen, Z., Hu, J., Zhang, Z., Jiang, S., Han, S., Yan, D., ... \& Zhang, Z. (2020b). Efficacy of hydroxychloroquine in patients with COVID-19: results of a randomized clinical trial. medrxiv. https://doi.org/10.1101/2020.03.22.20040758

Cheng, H. Y., Jian, S. W., Liu, D. P., Ng, T. C., Huang, W. T., \& Lin, H. H. (2020). Contact tracing assessment of COVID-19 transmission dynamics in Taiwan and risk at different exposure periods before and after symptom onset. JAMA Internal Medicine, 180(9), 1156-1163. https://doi.org/10.1001/jamainternmed.2020.2020

Du, Z., Xu, X., Wu, Y., Wang, L., Cowling, B. J., \& Meyers, L. A. (2020). Serial interval of COVID-19 among publicly reported confirmed cases. Emerging Infectious Diseases, 26(6), 1341. https://doi.org/10.3201/eid2606.200357

Fang, Y., Nie, Y., \& Penny, M. (2020). Transmission dynamics of the COVID-19 outbreak and effectiveness of government interventions: A data-driven analysis. Journal of Medical Virology, 92(6), 645-659. https://doi.org/10.1002/jmv.25750

Ferretti, L., Wymant, C., Kendall, M., Zhao, L., Nurtay, A., Abeler-Dörner, L., ... \& Fraser, C. (2020). Quantifying SARS-CoV-2 transmission suggests epidemic control with digital contact tracing. Science, 368(6491). https://doi.org/10.1126/science.abb6936

Gao, J., Tian, Z., \& Yang, X. (2020). Breakthrough: Chloroquine phosphate has shown apparent efficacy in treatment of COVID-19 associated pneumonia in clinical studies. Bioscience trends, 14(1), 72-73. https://doi.org/10.5582/bst.2020.01047

Gautret, P., Lagier, J. C., Parola, P., Meddeb, L., Mailhe, M., Doudier, B., ... \& Raoult, D. (2020a). Hydroxychloroquine and azithromycin as a treatment of COVID-19: results of an open-label non-randomized clinical trial. International Journal of Antimicrobial Agents, 56(1), 105949. https://doi.org/10.1016/j.ijantimicag.2020.105949

Gautret, P., Lagier, J. C., Parola, P., Meddeb, L., Sevestre, J., Mailhe, M., ... \& Raoult, D. (2020b). Clinical and microbiological effect of a combination of hydroxychloroquine and azithromycin in 80 COVID-19 patients with at least a six-day follow up: a pilot observational study. Travel Medicine and Infectious Disease, 34, 101663.

https://doi.org/10.1016/j.tmaid.2020.101663

Guan, W. J., Ni, Z. Y., Hu, Y., Liang, W. H., Ou, C. Q., He, J. X., ... \& Zhong, N. S. (2020). Clinical characteristics of coronavirus disease 2019 in China. New England Journal of Medicine, 382(18), 1708-1720. https://doi.org/10.1056/NEJMoa2002032
Gudbjartsson, D. F., Helgason, A., Jonsson, H., Magnusson, O. T., Melsted, P., Norddahl, G. L., ... \& Stefansson, K. (2020). Spread of SARS-CoV-2 in the Icelandic population. New England Journal of Medicine, 382(24), 2302-2315. https://doi.org/10.1101/2020.03.26.20044446

He, X., Lau, E. H., Wu, P., Deng, X., Wang, J., Hao, X., ... \& Leung, G. M. (2020). Temporal dynamics in viral shedding and transmissibility of COVID-19. Nature Medicine, 26(5), 672-675. https://doi.org/10.1038/s41591-020-0869-5

Hu, Z., Song, C., Xu, C., Jin, G., Chen, Y., Xu, X., ... \& Shen, H. (2020). Clinical characteristics of 24 asymptomatic infections with COVID-19 screened among close contacts in Nanjing, China. Science China Life Sciences, 63(5), 706-711. https://doi.org/10.1007/s11427-020-1661-4

Huang, C., Wang, Y., Li, X., Ren, L., Zhao, J., Hu, Y., ... \& Cao, B. (2020). Clinical features of patients infected with 2019 novel coronavirus in Wuhan, China. The Lancet, 395(10223), 497-506. https://doi.org/10.1016/S0140-6736(20)30183-5

Jin, S., \& Hu, W. (2021). Severity of COVID-19 and Treatment Strategy for Patient With Diabetes. Frontiers in Endocrinology, 12, 469. https://doi.org/10.3389/fendo.2021.602735

Juurlink, D. N. (2020). Safety considerations with chloroquine, hydroxychloroquine and azithromycin in the management of SARS-CoV-2 infection. CMAJ, 192(17), E450-E453. https://doi.org/10.1503/cmaj.200528

Lane, J. C., Weaver, J., Kostka, K., Duarte-Salles, T., Abrahao, M. T. F., Alghoul, H., ... \& Prieto-Alhambra, D. (2020). Risk of hydroxychloroquine alone and in combination with azithromycin in the treatment of rheumatoid arthritis: a multinational, retrospective study. The Lancet Rheumatology, 2(11), e698-e711. https://doi.org/10.1016/s2665-9913(20)30276-9

Lauer, S. A., Grantz, K. H., Bi, Q., Jones, F. K., Zheng, Q., Meredith, H. R., ... \& Lessler, J. (2020). The incubation period of coronavirus disease 2019 (COVID-19) from publicly reported confirmed cases: estimation and application. Annals of Internal Medicine, 172(9), 577-582. https://doi.org/10.7326/M20-0504

Lavezzo, E., Franchin, E., Ciavarella, C., CuomoDannenburg, G., Barzon, L., Del Vecchio, C., ... \& Crisanti, A. (2020). Suppression of COVID-19 outbreak in the municipality of Vo', Italy. MedRxiv. https://doi.org/10.1101/2020.04.17.20053157

Leung, N. H., Chu, D. K., Shiu, E. Y., Chan, K. H., McDevitt, J. J., Hau, B. J., ... \& Cowling, B. J. (2020). Respiratory virus shedding in exhaled breath and efficacy of face masks. Nature medicine, 26(5), 676-680. https://doi.org/10.1038/s41591-020-0843-2 
Li, R., Pei, S., Chen, B., Song, Y., Zhang, T., Yang, W., \& Shaman, J. (2020). Substantial undocumented infection facilitates the rapid dissemination of novel coronavirus (SARS-CoV-2). Science, 368(6490), 489-493. https://doi.org/10.1126/science.abb3221

Liang, L., \& Wu, P. (2020). There may be virus in conjunctival secretion of patients with COVID-19. Acta Ophthalmologica, 98(3), 223. https://doi.org/10.1111/aos.14413

Liu, Y., Ning, Z., Chen, Y., Guo, M., Liu, Y., Gali, N. K., ... \& Lan, K. (2020b). Aerodynamic analysis of SARS-CoV-2 in two Wuhan hospitals. Nature, 582(7813), $557-560$ https://doi.org/10.1038/s41586-020-2271-3

Liu, Y., Yan, L. M., Wan, L., Xiang, T. X., Le, A., Liu, J. M., ... \& Zhang, W. (2020a). Viral dynamics in mild and severe cases of COVID-19. The Lancet Infectious Diseases, 20(6), 656-657. https://doi.org/10.1016/S1473-3099(20)30232-2

Magagnoli, J., Narendran, S., Pereira, F., Cummings, T. H., Hardin, J. W., Sutton, S. S., \& Ambati, J. (2020). Outcomes of hydroxychloroquine usage in United States veterans hospitalized with Covid-19. Med, 1(1), 114-127. https://doi.org/10.1101/2020.04.16.20065920

Mahevas, M., Tran, V. T., Roumier, M., Chabrol, A., Paule, R., Guillaud, C., ... \& Costedoat, N. (2020). No evidence of clinical efficacy of hydroxychloroquine in patients hospitalized for COVID-19 infection with oxygen requirement: results of a study using routinely collected data to emulate a target trial. medrxiv. https://doi.org/10.1101/2020.04.10.20060699

McMichael, T. M., Clark, S., Pogosjans, S., Kay, M., Lewis, J., Baer, A., ... \& County, K. (2020). COVID-19 in a long-term care facility-King County, Washington, February 27-March 9, 2020. Morbidity and Mortality Weekly Report, 69(12), 339. https://doi.org/10.15585/mmwr.mm6912e1

Mizumoto, K., Kagaya, K., Zarebski, A., \& Chowell, G. (2020). Estimating the asymptomatic proportion of coronavirus disease 2019 (COVID-19) cases on board the Diamond Princess cruise ship, Yokohama, Japan, 2020. Eurosurveillance, 25(10), 2000180. https://doi.org/10.2807/1560-

7917.ES.2020.25.10.2000180

Ong, S. W. X., Tan, Y. K., Chia, P. Y., Lee, T. H., Ng, O. T., Wong, M. S. Y., \& Marimuthu, K. (2020). Air, surface environmental and personal protective equipment contamination by severe acute respiratory syndrome coronavirus 2 (SARS-CoV-2) from a symptomatic patient. JAMA, 323(16), 1610-1612. https://doi.org/10.1001/jama.2020.3227
Pan, Y., Zhang, D., Yang, P., Poon, L. L., \& Wang, Q. (2020a). Viral load of SARS-CoV-2 in clinical samples. The Lancet infectious diseases, 20, 411-412. https://doi.org/10.1016/S1473-3099(20)30113-4

Pan, X., Chen, D., Xia, Y., Wu, X., Li, T., Ou, X., ... \& Liu, J. (2020b). Asymptomatic cases in a family cluster with SARS-CoV-2 infection. The Lancet Infectious Diseases, 20(4), 410-411. https://doi.org/10.1016/S1473-3099(20)30114-6

Pan, L., Mu, M., Yang, P., Sun, Y., Wang, R., Yan, J., ... \& Tu, L. (2020c). Clinical characteristics of COVID19 patients with digestive symptoms in Hubei, China: a descriptive, cross-sectional, multicenter study. The American Journal of Gastroenterology, 115, 766-773. https://doi.org/10.14309/ajg.0000000000000620

Qian, G., Yang, N., Ma, A. H. Y., Wang, L., Li, G., Chen, X., \& Chen, X. (2020). COVID-19 transmission within a family cluster by presymptomatic carriers in China. Clinical Infectious Diseases, 71(15), 861-862. https://doi.org/10.1093/cid/ciaa316

Radonovich, L. J., Simberkoff, M. S., Bessesen, M. T., Brown, A. C., Cummings, D. A., Gaydos, C. A., ... \& Perl, T. M. (2019). N95 respirators vs medical masks for preventing influenza among health care personnel: a randomized clinical trial. JAMA, 322(9), 824-833. https://doi.org/10.1001/jama.2019.11645

Reinhard, A., Ikonomidis, C., Broome, M., \& Gorostidi, F. (2020). Anosmia and COVID-19. Revue Medicale Suisse, 16(691-2), 849-851. https://pubmed.ncbi.nlm.nih.gov/32348051/

Siddiqi, H. K., \& Mehra, M. R. (2020). COVID-19 illness in native and immunosuppressed states: a clinical-therapeutic staging proposal. The Journal of Heart and Lung Transplantation, 39(5), 405. https://doi.org/10.1016/j.healun.2020.03.012

Tang, W., Cao, Z., Han, M., Wang, Z., Chen, J., Sun, W., ... \& Xie, Q. (2020). Hydroxychloroquine in patients with mainly mild to moderate coronavirus disease 2019: open label, randomised controlled trial. BMJ, 369. https://doi.org/10.1101/2020.04.10.20060558

To, K. K. W., Tsang, O. T. Y., Leung, W. S., Tam, A. R., Wu, T. C., Lung, D. C., ... \& Yuen, K. Y. (2020). Temporal profiles of viral load in posterior oropharyngeal saliva samples and serum antibody responses during infection by SARS-CoV-2: an observational cohort study. The Lancet Infectious Diseases, 20(5), 565-574. https://doi.org/10.1016/S1473-3099(20)30196-1

Van Doremalen, N., Bushmaker, T., Morris, D. H., Holbrook, M. G., Gamble, A., Williamson, B. N., ... \& Munster, V. J. (2020). Aerosol and surface stability of SARS-CoV-2 as compared with SARSCoV-1. New England Journal of Medicine, 382(16), 1564-1567. https://doi.org/10.1056/NEJMc2004973 
Wang, W., Xu, Y., Gao, R., Lu, R., Han, K., Wu, G., \& Tan, W. (2020a). Detection of SARS-CoV-2 in different types of clinical specimens. JAMA, 323(18), 1843-1844. https://doi.org/10.1001/jama.2020.3786

Wang, Y., Liu, Y., Liu, L., Wang, X., Luo, N., \& Li, L. (2020b). Clinical outcomes in 55 patients with severe acute respiratory syndrome coronavirus 2 who were asymptomatic at hospital admission in Shenzhen, China. The Journal of Infectious Diseases, 221(11), 1770-1774. https://doi.org/10.1093/infdis/jiaa119

Wang, M., Cao, R., Zhang, L., Yang, X., Liu, J., Xu, M., ... \& Xiao, G. (2020c). Remdesivir and chloroquine effectively inhibit the recently emerged novel coronavirus (2019-nCoV) in vitro. Cell research, 30(3), 269-271. https://doi.org/10.1038/s41422-020-0282-0

Woelfel, R., Corman, V. M., Guggemos, W., Seilmaier, M., Zange, S., Mueller, M. A., ... \& Wendtner, C. (2020). Clinical presentation and virological assessment of hospitalized cases of coronavirus disease 2019 in a travel-associated transmission cluster. MedRxiv.

https://doi.org/10.1101/2020.03.05.20030502
Wu, X., Nethery, R. C., Sabath, B. M., Braun, D., \& Dominici, F. (2020). Exposure to air pollution and COVID-19 mortality in the United States. MedRxiv. https://doi.org/10.1101/2020.04.05.20054502

Xiao, A. T., Tong, Y. X., \& Zhang, S. (2020). Profile of RT-PCR for SARS-CoV-2: a preliminary study from 56 COVID-19 patients. Clinical Infectious Diseases, 71(16), 2249-2251. https://doi.org/10.1093/cid/ciaa460

Zhou, F., Yu, T., Du, R., Fan, G., Liu, Y., Liu, Z., ... \& Cao, B. (2020). Clinical course and risk factors for mortality of adult inpatients with COVID-19 in Wuhan, China: a retrospective cohort study. The Lancet, 395(10229), 1054-1062. https://doi.org/10.1016/S0140-6736(20)30566-3

Zou, L., Ruan, F., Huang, M., Liang, L., Huang, H., Hong, Z., ... \& Wu, J. (2020). SARS-CoV-2 viral load in upper respiratory specimens of infected patients. New England Journal of Medicine, 382(12), 1177-1179. https://doi.org/10.1056/NEJMc2001737 